\title{
Lixiviação de flazasulfuron e flumioxazin em dois diferentes latossolos ${ }^{1}$
}

\author{
Leaching of flazasulfuron and flumioxazin in two different soils
}

\author{
Robert Deuber ${ }^{2}$, Waldinei Pastre ${ }^{3}$; Andressa Barbosa Giusto ${ }^{3}$
}

\begin{abstract}
Resumo - Estudou-se a lixiviação dos herbicidas flazasulfuron (50 e $100 \mathrm{~g} \mathrm{ha}^{-1}$ ) e flumioxazin $\left(50 \mathrm{~g} \mathrm{ha}^{-1}\right)$ em um Latossolo Vermelho eutroférrico (LRe) e um Latossolo Vermelho amarelo distrófico (LVd), aplicando chuvas simuladas de 50 e $100 \mathrm{~mm}$. Amostras de cada solo foram colocadas em tubos de PVC com $50 \mathrm{~cm}$ de altura e $10 \mathrm{~cm}$ de diâmetro, previamente cortados ao meio, longitudinalmente e emendados com fita adesiva. Os herbicidas foram aplicados com pulverizador de $\mathrm{CO}_{2}$ sobre a superfície dos tubos. As chuvas foram aplicadas de $10 \mathrm{em} 10 \mathrm{~mm}$, gotejados por meio de uma chapa crivada. Havia uma testemunha que apenas recebeu as chuvas e todos os tratamenos foram repetidos quatro vezes em um delineamento totalmente casualizado. Após a aplicação das chuvas os tubos foram seccionados longitudinalmente, semeando-se pepino (Cucumis sativus) e aveia (Avena sativa) ao longo dos mesmos, como plantas-teste. Aos 7, 11, 14 e 23 dias após a semeadura, foram feitas avaliações visuais de sintomas nessas plantas, registrando-se até que profundidade do perfil eram perceptíveis. Não houve nenhuma resposta com a aveia para os dois herbicidas. Flazasulfuron a $50 \mathrm{~g} \mathrm{ha}^{-1}$ no solo LRe lixiviou até $6,5 \mathrm{~cm}$ com $50 \mathrm{~mm}$ de chuva e até $10 \mathrm{~cm}$ com $100 \mathrm{~mm}$. No solo LVd atingiu $3,6 \mathrm{~cm}$ com $50 \mathrm{~mm}$ de chuva e detectado até $1,1 \mathrm{~cm}$ com $100 \mathrm{~mm}$. Na dose de $100 \mathrm{~g} . h a^{-1}$, no LRe atingiu $6,2 \mathrm{~cm}$ com 50 $\mathrm{mm}$ e $10,5 \mathrm{~cm}$ com $100 \mathrm{~mm}$. No LVd sintomas foram detectados apenas até $2,0 \mathrm{~cm}$ com ambas as chuvas. O flumioxazin apresentou pouca lixiviação em ambos os solos, sendo os sintomas observados apenas até $2,7 \mathrm{~cm}$ no LRe e, até $2,0 \mathrm{~cm}$ no LVd. As diferenças de lixivação observadas foram dependentes dos teores de matéria orgânica dos solos.
\end{abstract}

Palavras-chave: Comportamento de herbicidas, resíduos no solo, precipitação.

Abstract - Flazasulfuron is recommended for sugarcane and flumioxazin for soybean and other leguminous crops in Brazil. Due to the importance of these crops the herbicides may be widely used and there is a need to know their behavior in the cultivated soils. In order to study the leaching of these compounds, an experiment was carried out in greenhouse with two soils: a sandy loam Typic Hapludox (LVd) with $22 \mathrm{~g} \mathrm{dm}^{-3}$ of organic matter and a clay Rhodic Eutrudox (LRe) with 13 g.dm ${ }^{-3}$ of organic matter. Flazasulfuron was applied at 50 and $100 \mathrm{~g}^{-h^{-1}}$ and flumioxazin at $50 \mathrm{~g} \mathrm{ha}^{-1}$. The soils were put in $50 \mathrm{~cm}$ high PVC columns, with $10 \mathrm{~cm}$ diameter. The herbicides were applied with a $\mathrm{CO}_{2}$ sprayer over the surface of the columns. Rains of 50 and $100 \mathrm{~mm}$ were dripped over the surface with perforated recipients in amounts of $10 \mathrm{~mm}$ each time. All treatments had four replicates. After this, the columns were cut longitudinally in halves and

\footnotetext{
${ }^{1}$ Trabalho parcialmente apresentado no V International Weed Science Congress, em Vancouver, Canada, 2008.

2 Instituto Agronômico de Campinas, rdeuber@iac.sp.gov.br;

${ }^{3}$ Pós-Graduação do IAC - Àrea de Produção Agrícola
} 
Cucumis sativus and Avena sativa were sown all along as test-plants. Visual evaluations of herbicide symptoms were done at 7, 11, 14 and 23 days after sowing, measuring the extent of damaged plants along the columns. Only C.sativus showed sensibility to the herbicides. Flazasulfuron at $50 \mathrm{~g}$, in $\mathrm{LRe}$, leached to $6,5 \mathrm{~cm}$ with $50 \mathrm{~mm}$ of rain and to $10 \mathrm{~cm}$ with $100 \mathrm{~mm}$. In the LVd soil, it reached $3,6 \mathrm{~cm}$ with $50 \mathrm{~mm}$ and $1,1 \mathrm{~cm}$ with $100 \mathrm{~mm}$. At $100 \mathrm{~g}$, in LRe it leached $6,2 \mathrm{~cm}$ with $50 \mathrm{~mm}$ and $10,5 \mathrm{~cm}$ with $100 \mathrm{~mm}$. In the LVd it reached no more than 2,0 $\mathrm{cm}$ with both rains. Flumioxazin showed very little leaching in both soils, being the symptons detected in no more than 2,7 cm in the LRe and 2,0 cm in the LVd. Symptons of the herbicides were detected in the bottom of the columns, showing that some amount was leached through the entire column of soil. The differences observed between the soils may were related to the organic mater content of the soils.

Key words: herbicide behavior, soil characteristics, residues in soil, precipitation

\section{Introdução}

O herbicida flazasulfuron é recomendado para a cultura da cana-de-açúcar e o flumioxazin, para a cultura da soja e outras leguminosas. Tendo em vista o grande potencial de utilização, é importante conhecer a dinâmica desses compostos, principalmente os aspectos referentes a movimentação desses herbicidas no perfil do solo. Atualmente são poucos os trabalhos relacionados com $\mathrm{O}$ comportamento desses herbicidas no solo.

O flazasulfuron é um composto relativamente estável, apresentando mineralização de 2 a 5\% após 100 dias (Europa, 2003). A meia-vida desse composto é de, aproximadamente 30 dias, com persistência maior quando o $\mathrm{pH}$ é mais elevado e a sua mobilidade tende a aumentar no solo com a elevação do pH (EUA, 2007). O flumioxazin apresenta meia vida de 27 a 38 dias em condições de campo e com lixiviação muito dependente dos teores de argila, sendo que maiores concentrações foram encontradas na faixa de zero a $7,5 \mathrm{~cm}$ de profundidade, utilizando-se lisímetros e medição da radioatividade (Australia, 2002). Apresenta mobilidade moderada nos solos, não sendo encontrado, em geral, em profundidades além de 7,5 cm (EUA, 2001).
Oliveira et al. (2005) observaram que a hidrólise do flazasulfuron foi maior em ambientes com menores valores de $\mathrm{pH}$ e em temperaturas mais elevadas, sendo que a sorção desse herbicida apresentou correlação positiva e significativa com os teores de carbono orgânico, silte, CTC e umidade do solo. Estes autores constataram, ainda, que o pH do solo apresenta correlação inversamente proporcional à sorção do flazasulfuron, indicando maior risco de lixiviação com a elevação do $\mathrm{pH}$.

Em relação ao flumioxazin, Oliveira et al. (1999) verificaram que este herbicida lixiviou até $5 \mathrm{~cm}$ em um solo Podzólico Vermelho-Amarelo câmbico (PVc) e até $10 \mathrm{~cm}$ em um Latossolo Vermelho-amarelo (LVm), com chuvas simuladas de 45 e $90 \mathrm{~mm}$. Segundo Alister et al (2008), trabalhando em condições de campo no Chile em quatro localidades diferentes, o flumioxazin apresentou $\mathrm{DT}_{50}$ de 10 a 32 dias, dependendo da precipitação e do $\mathrm{pH}$. Também verificaram lixiviação de $45 \%$ do herbicida aplicado na superfície até abaixo de $15 \mathrm{~cm}$ nos solos estudados.

Dentro deste contexto o trabalho objetivou avaliar o potencial de lixiviação de flazasulfuron e de flumioxazin, utilizando amostras de solos Latossolo Vermelho 
eutroférrico e Latossolo Vermelho amarelo distrófico em colunas.

\section{Material e métodos}

Amostras de um Latossolo Vermelho eutroférrico (LRe) e de um Latosso Vermelho amarelo distrófico (LVd) foram coletadas em áreas localizadas no Centro Experimental de Campinas, do IAC, com as características apresentadas na Tabela 1.
As amostras de terra foram coletadas a profundidade de 0 a $40 \mathrm{~cm}$ nos dois locais, secadas ao ar e passadas em peneira de malha 50. A seguir, o material foi colocado em tubos de $\mathrm{PVC}$, de $10 \mathrm{~cm}$ de diâmetro e $50 \mathrm{~cm}$ de altura, previamente secciondas longitudinalmente e emendadas com fita adesiva.

Tabela 1. Características físicas e químicas dos solos estudados no experimento de lixiviação de herbicidas em colunas. IAC, 2002.

\begin{tabular}{ccccccccc}
\hline SOLO & Argila & Silte & $\begin{array}{c}\text { Areia } \\
\text { total }\end{array}$ & $\begin{array}{c}\text { Areia } \\
\text { grossa }\end{array}$ & $\begin{array}{c}\text { Areia } \\
\text { fina }\end{array}$ & $\begin{array}{c}\text { Matéria } \\
\text { orgânica }\end{array}$ & $\mathbf{p H}$ & Classificação textural \\
\hline & & & $\mathbf{g . k g}^{-1}$ & & & $\mathbf{g . d m}{ }^{-3}$ & $\mathbf{C a C l}_{\mathbf{2}}$ & \\
\hline $\mathrm{LRe}^{1}$ & 513 & 130 & 358 & 226 & 132 & 13 & 5,2 & Argilosa \\
\hline $\mathrm{LVd}^{2}$ & 288 & 99 & 614 & 405 & 208 & 22 & 4,6 & Franco-argilo-arenosa \\
\hline
\end{tabular}

Obs.: ${ }^{1}$ Latossolo Vermelho eutroférrico; ${ }^{2}$ Latossolo Vermelho escuro distrófico.

O fundo dos tubos foi fechado com um tecido resistente e de malha bem fechada, para evitar escoamento de terra quando da percolação da água através das colunas. Após o acondicionamento das amostras, em cada tubo adicionou-se água até que houvesse perfeita acomodação da terra, ficando a superfície um $\mathrm{cm}$ abaixo do bordo superior.
Os herbicidas estudados foram: flazasulfuron $\{1-(4,6-$ dimetoxipirimidina-2-il3-(3-trifluorometil-2-piridilsulfonil) uréia\}; e flumioxazin \{7-fluoro-6-[(3.4.5.6tetrahidro)ftalimida]-4-(2-propinil)-1,4benzona-zino-3-(2H)-ona $\}$. Os tratamentos e as características dos herbicidas são apresentados na Tabela 2.

Tabela 2. Doses e características dos herbicidas estudados no experimento de lixiviação em colunas.

\begin{tabular}{|c|c|c|c|c|}
\hline $\begin{array}{l}\text { Herbicida e dose } \\
\text { i.a.ha }\end{array}$ & Formulação & pKa & $\begin{array}{c}\text { Solubilidade } \\
\text { em água a } 25^{\circ} \mathrm{C}\end{array}$ & $\begin{array}{c}\text { Classe de } \\
\text { Solubilidade }\end{array}$ \\
\hline Flazasulfuron $50 \mathrm{~g}$ & \multirow{2}{*}{$\begin{array}{l}\text { granulado } \\
\text { dispersível } \\
250 \mathrm{~g} . \mathrm{kg}^{-1}\end{array}$} & 4,37 & \multirow{2}{*}{$\begin{array}{c}0,027 \mathrm{mg} \cdot \mathrm{ml}^{-1} \text { a } \mathrm{pH} 5,0 \\
\left(2,1 \text { g.L } \mathrm{L}^{-1} \text { a } \mathrm{pH} 7,0\right)\end{array}$} & \multirow{2}{*}{$\begin{array}{c}\text { baixa } \\
\text { (muito elevada) }\end{array}$} \\
\hline Flazasulfuron $100 \mathrm{~g}$ & & & & \\
\hline Flumioxazin $50 \mathrm{~g}$ & $\begin{array}{l}\text { pó molhável } \\
500 \text { g.kg-1 }\end{array}$ & não dissocia & $1,79 \mathrm{mg} \cdot \mathrm{L}^{-1}$ & muito baixa \\
\hline
\end{tabular}


A testemunha correspondeu, apenas a aplicação da água, da mesma maneira que nos tratamentos com herbicida. Cada tratamento foi repetido quatro vezes e os tubos ficaram em disposição totalmente casualizada dentro de casa-de-vegetação, sem controle das condições climáticas.

As aplicações dos herbicidas foram realizadas com pulverizador de $\mathrm{CO}_{2}$, a pressão constante de $2,1 \mathrm{~kg} . \mathrm{cm}^{-2}$, munido de quatro bicos leque 80.02 , espaçados de $0,50 \mathrm{~m}$, com consumo equivalente a 400 litros de calda por hectare. Os tubos foram dispostos sobre o solo, dentro da faixa de aplicação, que foi realizada passando-se a barra a $0,50 \mathrm{~m}$ acima da superfície das colunas.

As simulações de precipitação foram de 50 e de $100 \mathrm{~mm}$ de água, aspergidos sobre a superfície com recipientes com fundo crivado, de $10 \mathrm{em} 10 \mathrm{~mm}$, até se atingir a quantidade desejada. Após sete dias da percolação final, os tubos foram cuidadosamente cortados ao meio, ao longo da fita adesiva, para não movimentar a terra e, a seguir, colocados longitudinalmente sobre uma bancada. Em uma das partes semeou-se pepino (Cucumis sativa L. var. Safira) e na outra, semeou-se aveia-branca (Avena sativa L.).

As avaliações visuais da lixiviação de cada herbicida foram realizadas aos $7,11,14 \mathrm{e}$ 23 dias após a semeadura, medindo-se a distância, a partir da superfície, até aonde se manifestavam sintomas de fitotoxicidade nas plantas-teste. Aos 11, 14 e 23 dias também se mediu a distância, a partir do fundo, em que se verificavam sintomas de fitotoxicidade, para avaliar a presença dos herbicidas que tivessem lixiviado até a base da coluna.

Os resultados foram submetidos à análise da variância, combinando-se os diversos fatores para verificar as possíveis interações entre os êles.

\section{Resultados e discussão}

A avaliação da profundidade de lixiviação deveria ser realizada com a indicação de duas plantas teste, pepino e aveia, para melhor detalhamento da distribuição dos herbicidas no perfil da coluna. Essa detecção diferenciada ocorre em função dos variados níveis de sensibilidade das diferentes espécies vegetais. Como a aveia não apresentou sensibilidade suficiente para os herbicidas estudados, em nenhum dos tratamentos aplicados, as avaliações visuais foram feitas apenas com pepino.

Os resultados de detecção da lixiviação de flazasulfuron pelas plantas de pepino nos dois tipos de solo estão apresentados na Tabela 3 e nas Figuras 2 e 3. A Figura 1 ilustra a disposição dos tubos cortados com a presença das plantas de pepino.

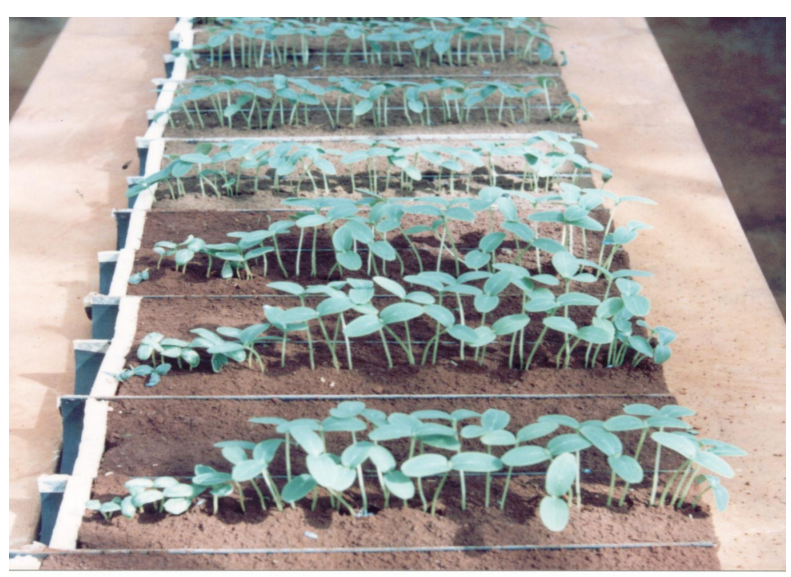

Figura 1. Vista dos tubos cortados com tratamento de flazasulfuron a 100 g.ha ${ }^{-1}$, e os sintomas em plantas de pepino, em primeiro plano, com precipitação simulada de $100 \mathrm{~mm}$ de chuva, em Latossolo Vermelho eutrófico, aos 23 dias após a aplicação dos tratamentos. 
Tabela 3. Profundidade de lixiviação dos herbicidas flazasulfuron e flumioxazin em colunas de 50 cm, em dois diferentes tipos de solo, em casa-de-vegetação. 2004.

\begin{tabular}{|c|c|c|c|c|c|c|c|c|c|}
\hline \multirow{2}{*}{$\frac{\text { Tratamentos }}{\text { Dose.i.a.ha }^{-1}}$} & \multirow[b]{2}{*}{$\begin{array}{c}\text { Chuva } \\
\mathrm{mm}\end{array}$} & \multicolumn{2}{|c|}{7 DIAS } & \multicolumn{2}{|c|}{11 DIAS } & \multicolumn{2}{|c|}{14 DIAS } & \multicolumn{2}{|c|}{23 DIAS } \\
\hline & & $\operatorname{LRe}^{(1)}$ & $\mathrm{LVd}^{(2)}$ & LRe & LVd & LRe & LVd & LRe & LVd \\
\hline & & \multicolumn{8}{|c|}{$\mathrm{cm}$} \\
\hline Flazasulfuron & 50 & $4,5 \mathrm{a} \mathrm{AB}$ & & $5,87 \mathrm{aB}$ & $2,85 \mathrm{bA}$ & $6,37 \mathrm{aAH}$ & $3,62 \mathrm{bA}$ & 6,62 a B & $3,12 \mathrm{~b} \mathrm{~A}$ \\
\hline Flazasulfuron & 100 & $4,0 \mathrm{a} A B$ & $1,15 \mathrm{bAB}$ & $9,62 \mathrm{aA}$ & $1,62 \mathrm{bAB}$ & $9,87 \mathrm{aA}$ & $1,62 \mathrm{~b} \mathrm{~B}$ & 10,0 a A & $1,12 \mathrm{~b} \mathrm{~B}$ \\
\hline Flazasulfuron & 50 & $5,7 \mathrm{a} A B$ & $1,62 \mathrm{~b} \mathrm{AB}$ & $7,00 \mathrm{aAB}$ & $1,87 \mathrm{bAB}$ & $7,12 \mathrm{aAH}$ & $2,25 \mathrm{bAB}$ & 6,25 a $B$ & $1,75 \mathrm{bAB}$ \\
\hline Flazasulfuron & 100 & 10,7 a A & $1,0 \mathrm{~b} \mathrm{AB}$ & $10,37 \mathrm{aA}$ & $1,75 \mathrm{bAB}$ & $10,87 \mathrm{aA}$ & $1,75 \mathrm{bB}$ & $10,5 \mathrm{aA}$ & $2,00 \mathrm{bAB}$ \\
\hline Flumioxazin & 50 & $1,37 \mathrm{aC}$ & $1,37 \mathrm{aAB}$ & $1,37 \mathrm{aC}$ & $1,25 \mathrm{aAB}$ & $2,50 \mathrm{aC}$ & $2,25 \mathrm{aB}$ & 2,50 a C & $2,00 \mathrm{bAB}$ \\
\hline Flumioxazin & 100 & $0,37 \mathrm{aC}$ & $0,75 \mathrm{aB}$ & $1,62 \mathrm{aC}$ & $1,00 \mathrm{aB}$ & $2,25 \mathrm{aC}$ & 1,50 a B & 2,75 a C & $1,35 \mathrm{bB}$ \\
\hline $\begin{array}{ll}\text { C.V. } & 12,79 \% \\
\end{array}$ & & & & & & & & & \\
\hline
\end{tabular}

Obs: ${ }^{1}$ Latossolo Vermelho eutrófico; ${ }^{2}$ Latossolo Vermelho escuro distrófico. Letras minúsculas nas linhas indicam diferenças significativas entre solos ao nível de 5\% pelo teste de Duncan em cada época e, maiúsculas nas colunas diferenças de $5 \%$ entre todos os tratamentos, em cada época.

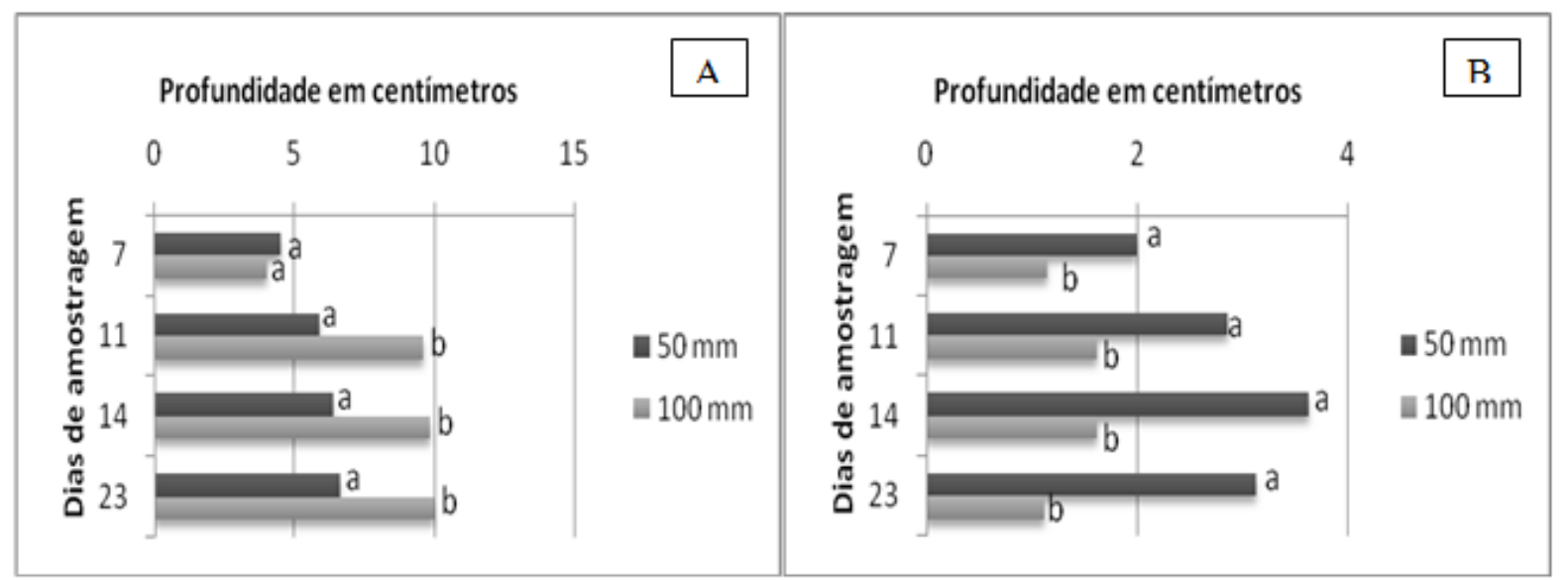

Figura 2. Lixiviação de flazasulfuron em $\operatorname{LRe}(\mathrm{A})$ e $\mathrm{LVd}(\mathrm{B})$, na dose de $50 \mathrm{~g} \cdot \mathrm{ha}^{-1}$ com precipitações simuladas de 50 e $100 \mathrm{~mm}$, avaliadas pela fitotoxicidade em Cucumis sativa L. Var. Safira, em diferentes épocas após a semeadura. Letras diferentes no final das colunas indicam diferença ao nível de $5 \%$ entre os regimes de chuva pelo teste de Duncan, em cada época de amostragem.

.A lixiviação do flazasulfuron foii significativamente diferente para os dois solo para todos os tratamentos $\mathrm{e}$ todas as amostragemns (Tabela 3). No solo LRe a lixiviação alcançou $1030 \mathrm{~cm}$ com $100 \mathrm{~mm}$ de chuva, para a dose de 50 g.ha ${ }^{-1}$ e $11 \mathrm{~cm}$ para 100 $\mathrm{g}$ (Figuras 2 e 3 ). No solo LVd, a profundidade máxima alcançada foi de $3,0 \mathrm{~cm}$ aproximadamente (Figura 3).

A diferença observada deve ser atribuída ao teor de matéria orgânica, que foi de $22 \mathrm{~g} . \mathrm{dm}^{-3}$ no solo LRe e de 13 g.dm ${ }^{-3}$ no LVd. Considerando-se a composição das argilas, segundo Melfi et al. (1966), o solo LRe contém caulinita e hematita e o solo LVd apenas caulinita, que é argila de baixa capacitade de sorção. Em trabalho anterior, com os mesmos solos, concluiu-se que a hematita não adsorvia o metribuzin (Paulo et al., 1979) o que poderia também ocorrer para a flazasulfuron que é um herbicida de caráter ácido. Dessa forma, o 
componente argila de ambos os solos apresentou pouca retenção do herbicida.
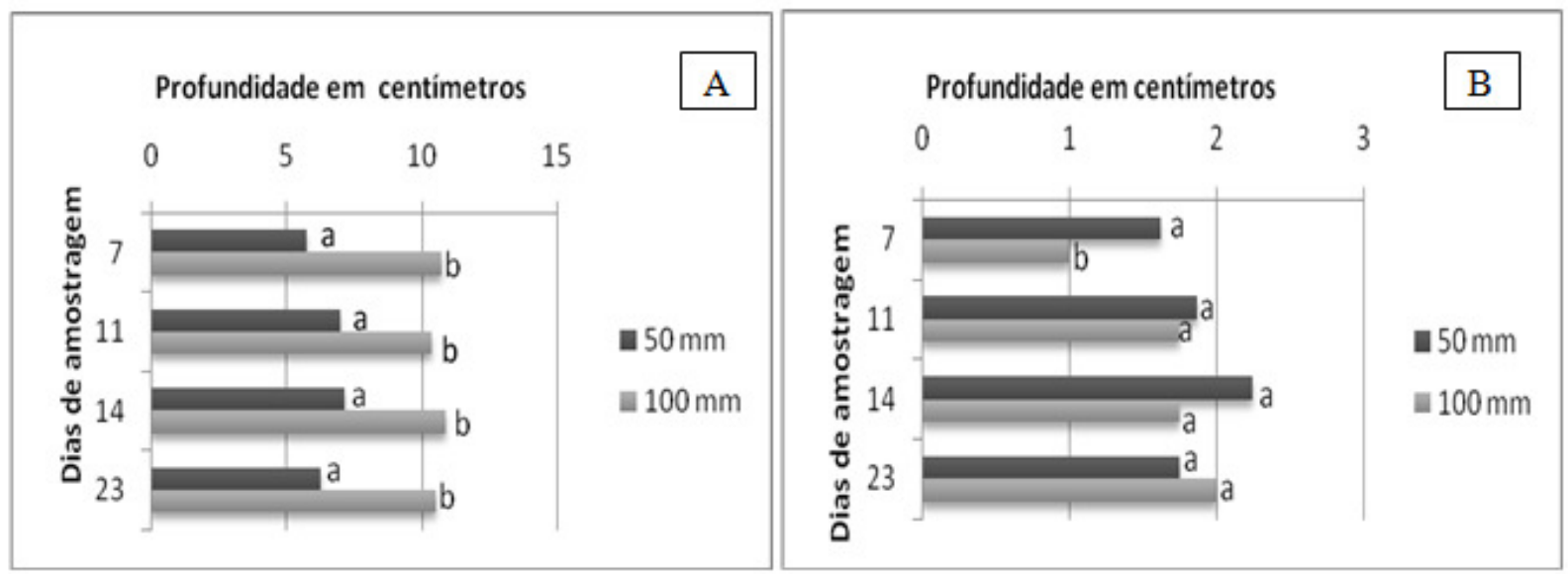

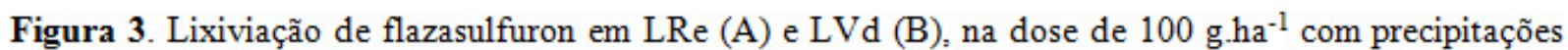
simuladas de 50 e $100 \mathrm{~mm}$, avaliadas por meio da fitotoxicidade em Cucumis sativa $\mathrm{L}$. Var. Safira, em diferentes épocas após a semeadura. Letras diferentes no final das colunas indicam diferença ao nível de $5 \%$ entre os regimes de chuva pelo teste de Duncan, em cada época de amostragem.

A menor lixiviação detectada no solo LVd com $100 \mathrm{~mm}$ de chuva pode estar relacionada ao fato de que uma parte do flazasulfuron ter sido lixiviada através do perfil todo, ficando mais concentrada na parte superior e bastante diluída ao longo da coluna, não sendo a concentração presente na parte intermediária, suficiente para ser detectada pelo pepino. Essa diferença foi pronunciada com a dose de $50 \mathrm{~g} / \mathrm{ha}$ mas, não foi verificada com 100 g. Observou-se durante a realização do estudo que ocorriam sintomas do herbicida no pepino na parte inferior do tubo (Tabela 4) nos dois tipos de solo, indicando que esse arrastamento ocorreu até os $50 \mathrm{~cm}$ de profundidade.

herbicida apresentou correlação positiva e significativa com os teores de carbono orgânico, silte, CTC e umidade do solo. Maiores valores de $\mathrm{pH}$ do solo reduziram a sorção do flazasulfuron, indicando maior risco de lixiviação, o que concorda com os resultados aqui obtidos, em que maior teor de matéria orgânica reduziu a lixiviação.
O valor do pKa do flazasulfuron é considerado médio e indica fraca retenção aos colóides de argila mas, podendo ser mais retido pela matéria orgânica. A solubilidade desse composto é considerada baixa a $25^{\circ} \mathrm{C}$ mas, pode ser muito elevada com o aumento do $\mathrm{pH}$ (Rodrigues \& Almeida, 2005). Como o pH do solo LRe é um pouco superior ao do LVd, podia-se esperar alguma diferença de lixiviação entre os dois solos por essa razão, além do teor de matéria orgânica

Oliveira et al. (2005) observaram em seus estudos que a hidrólise do flazasulfuron foi maior para menores valores de $\mathrm{pH}$ e em temperaturas mais elevadas. A sorção desse

A análise estatística revelou diferenças significativas de lixiviação para tipo de solo nas duas doses do flazasulfuron (Tabela 3), sendo em LRe sempre maior que em LVd, o que pode ser explicado pelo teor de matéria orgânica maior no LVd. Comparando-se as duas precipitações houve diferença entre ambas para a dose de $50 \mathrm{~g}$ nos dois solos e para $100 \mathrm{~g}$ 
no LRe (Figuras 2 e 3) mas, não, para a de 100 g.ha ${ }^{-1}$, no solo LVd (Figura 3B).

compostos estudados, diferindo significativamente dos tratamentos com

O herbicida flumioxazin apresentou as menores taxas de lixiviação dos dois flazasulfuron, tanto para solos quanto para chuvas (Tabela 3 e Figura 4).

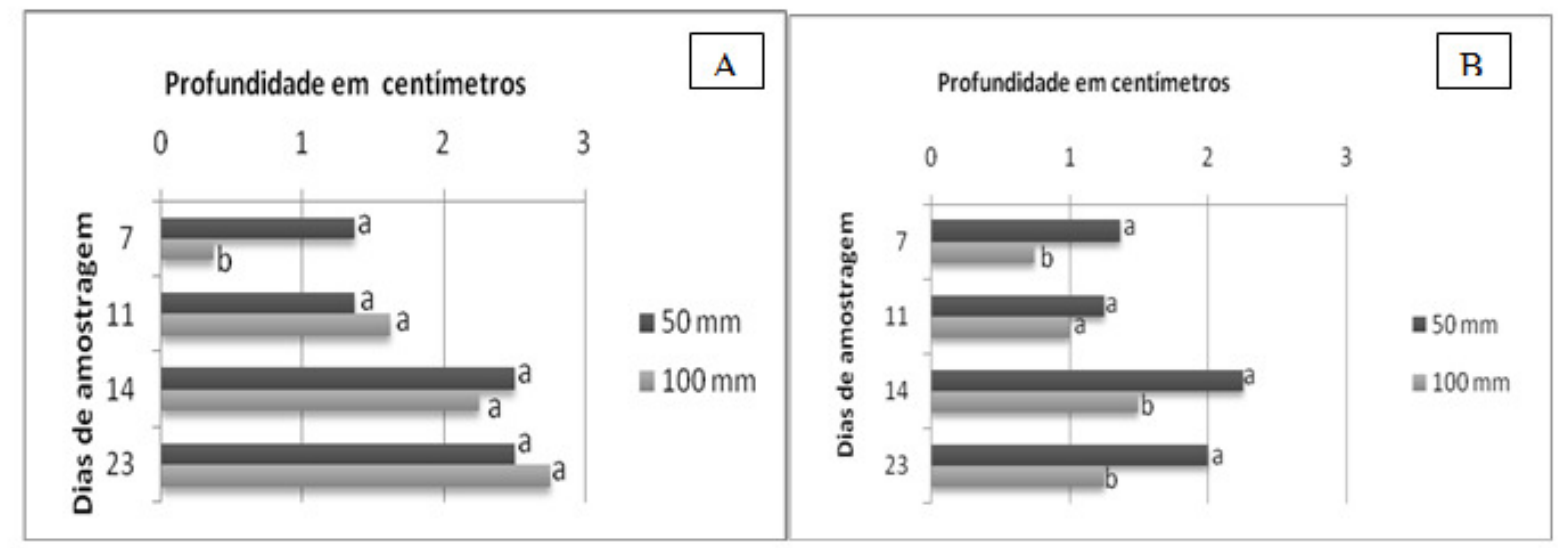

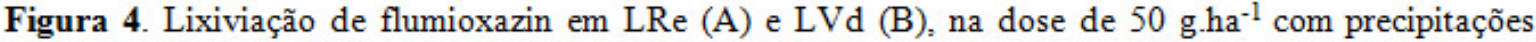
simuladas de 50 e $100 \mathrm{~mm}$, avaliada por meio da fitotoxicidade em Cucumis sativa L. Var. Safira, em diferentes épocas após a semeadura. Letras diferentes no final das colunas indicam diferença ao nível de $5 \%$ entre os regimes de chuva pelo teste de Duncan, em cada época de amostragem.

A profundidade máxima detectada pelas plantas de pepino foi de $2,75 \mathrm{~cm}$, no solo LRe, com $100 \mathrm{~mm}$ de chuva. Não houve diferenças significativas entre os dois solos nem entre as duas quantidades de chuva para lixiviação detectada desse herbicida (Tabela 3). Em estudo semelhante, Oliveira et al. (1999) trabalhando com chuvas de 45 e $90 \mathrm{~mm}$, encontraram lixiviação de até $5 \mathrm{~cm}$ em solo Podzólico Vermelho-amarelo câmbico (PVc), argiloso com elevado teor de matéria orgânica e de até $10 \mathrm{~cm}$ em solo Latossolo Vermelhoamarelo $(\mathrm{LVm})$, arenoso com baixo teor de matéria orgânica. Esses resultados foram maiores que os encontrados neste trabalho, sendo que as diferenças podem ter ocorrido devido à composição das argilas e da matéria orgânica. Por outro lado, Alister et al. (2008) observaram que, em condições de campo no Chile, o flumioxazin lixiviou em $45 \%$, além dos $15 \mathrm{~cm}$ de profundidade, atingindo, no máximo $45 \mathrm{~cm}$ do perfil. Esses resultados também diferem dos aqui encontrados, quanto à concentração nas camadas superiores mas, concordam com o fato de haver lixiviação até camadas inferiores, uma vez que foi verificada a presença do herbicida na base das colunas, com $50 \mathrm{~cm}$.

A lixiviação de flumioxazin foi similar a do flazasulfuron: parte do herbicida foi arrastada através dos tubos, sendo detectado na parte inferior. Para a chuva de $50 \mathrm{~mm}$ no solo LRe, o herbicida se concentrou ou longo dos $5,75 \mathrm{~cm}$ inferiores e, para $100 \mathrm{~mm}$ de chuva, em apenas $1,75 \mathrm{~cm}$. No solo LVd foi detectado apenas em 1,25 a 1,50 cm para as chuvas de 100 e 50, respectivamente (Tabela 4). Em função de sua não dissociação em água, o flumioxazin foi bastante lixiviado, em todas as condições, com possível perda além dos $50 \mathrm{~cm}$ das colunas. Apenas as plantas de pepino foram suficientemente sensíveis para detectá-lo.

Pode-se inferir que, para estudos dessa natureza, com precipitações elevadas, como as aqui aplicadas, o perfil de solo deverá ser maior, talvez de 80 a $100 \mathrm{~cm}$, para avaliar com 
mais precisão até onde pode ocorrer a se deve esperar problemas de efeitos residuais lixiviação. para culturas subsequentes, opinião

As baixas concentrações detectadas ao concordante à de Alister et al. (2008) para flumioxazin em solos do Chile. longo dos perfis dos solos, para os dois herbicidas estudados, permitem inferir que não Tabela 4. Detecção da presença dos herbicidas na parte inferior dos tubos por Cucumis sativus L. Var Sativa, no experimento de lixiviação em casa-de-vegetação. Campinas, SP. 2004.

\begin{tabular}{lllllll}
\hline \multicolumn{1}{c}{ TRATAMENTOS } & AOS 11 DAS & \multicolumn{2}{c}{ AOS 14 DAS } & \multicolumn{2}{c}{ AOS 23 DAS } \\
\hline \multicolumn{1}{c}{ Herbicidas e precipitações } & LRe & LVd & LRe & LVd & LRe & LVd \\
\hline & \multicolumn{7}{c}{ centímetros } \\
\hline Flazasulfuron a $50 \mathrm{~g} / \mathrm{ha} \mathrm{com} 50 \mathrm{~mm}$ & 3,00 & 1,25 & 2,75 & 3,62 & 3,00 & 3,00 \\
\hline Flazasulfuron a $50 \mathrm{~g} / \mathrm{ha}$ com $100 \mathrm{~mm}$ & 2,75 & 1,12 & 3,50 & 1,37 & 3,50 & 1,02 \\
\hline Flazasulfuron a $100 \mathrm{~g} /$ ha com $50 \mathrm{~mm}$ & 1,62 & 0,75 & 1,37 & 1,25 & 1,75 & 1,00 \\
\hline Flazasulfuron a $100 \mathrm{~g} / \mathrm{ha} \mathrm{com} 100 \mathrm{~mm}$ & 2,75 & 2,00 & 2,75 & 2,00 & 2,75 & 1,25 \\
\hline Flumioxazin a $50 \mathrm{~g} / \mathrm{ha} \mathrm{com} 50 \mathrm{~mm}$ & 4,75 & 0,75 & 4,50 & 1,33 & 5,75 & 1,50 \\
\hline Flumioxazin a $50 \mathrm{~g} / \mathrm{ha} \mathrm{com} 100 \mathrm{~mm}$ & 0,00 & 0,00 & 1,50 & 1,25 & 1,75 & 1,25 \\
\hline
\end{tabular}

DAS = dias após a semeadura $; \mathrm{LRe}=$ Latossolo Vermelho eutrófico; $\mathrm{LEd}=$ Latossolo vermelho escuro distrófico.

\section{Conclusões}

Flazasulfuron apresentou maior grau de lixiviação do que o flumioxazin no solo LRe e de forma semelhante no solo LVd;

As diferenças de lixivação observadas foram fortemente correlacionadas ao teor de matéria orgânica dos solos;

A concentração dos herbicidas foi baixa em todas as partes dos tubos, uma vez que a aveia não mostrou qualquer sintoma de intoxicação.

\section{Agradecimento}

Os autores agradecem à Pesquisadoracientífica Maria do Carmo de Salvo Soares Novo pela orientação na análise estatística dos dados.

\section{Referências}

ALISTER, C.; ROJAS, S.; GOMEZ, P.; KOGAN, M. Dissipation and movement of flumioxazin in soil of four field sites in Chile. Pest Management Sci,. v.64, n.5, p.579-583, 2008.

AUSTRÁLIA. Australian Pesticide and Veterinarian Medicines Authority. Evaluation of the new active flumioxazin in the product Pledge 500WG. 49 p. 2002. In: http://www.opuma.gov.au/ publications/downloads/prsflum.pdf._Acessado em 30 de março de 2009.

ESTADOS UNIDOS. Environmental Protection Agency. Office of Prevention, Pesticides and Toxic substances - Flumioxazin. 2001. In: http://www.epa.gov/opprd001/factsheets/flumioxazi n.pdf. Acessado em 10 de março de 2009.

ESTADOS UNIDOS. Environmental Protection Agency. Office of Prevention, Pesticides and Toxic Substances - Flazasulfuron. 2007. In: http://www.epa.gov/oppr001/factsheets/flazasulfuro n.pdf. Acessado em 10 de março de 2009.

EUROPA. Europa Council. 2007. In: http://www.ec.europa.eu/food/plant/protection/eval uation/newactive/flazasulfuron.pdf. Acessado em 15 de março de 2009.

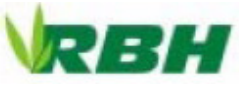


MELFI, A.J.; GIRARDI, V.A.V.; MONIZ, A.C. Mineralogia dos solos da Estação Experimental "Theodureto de Camargo" em Campinas. Bragantia, v.25, n.1, p.9-30, 1966.

OLIVEIRA, M.F. et al. Lixiviação de flumioxazin e metribuzin em dois solos em condições de laboratório. Planta Daninha, v.17, n.2, p.207-215, 1999.

OLIVEIRA, M.F et al. Sorção e hidrólise do herbicida flazasulfuron. Planta Daninha, v 23, n.1, p.101-113, 2005.

PAULO, E. M.; SIGNORI, L.H.; DEUBER, R. Lixiviação de metribuzin, oxadiazon e bromacil em dois tipos de solos. Planta Daninha, v.2, n.2, p.111-115, 1979.

RODRIGUES, B.N.; ALMEIDA, F.S. Guia de herbicidas. $5^{\text {a }}$ Edição. Londrina. Edição dos Autores, 2005. 591p.

WSSA - Weed Science Society of America. Herbicide Handbook. $8^{\mathrm{a}}$ Ed. Lawrence, 2002. 493p. 
Rev. Bras. Herb., v.8, n.1, p.27-36, jan./abr. 2009 